\title{
Development of multiwavelength- range fine-resolution spectrometer for hydrogen emissions and its application to large helical device periphery plasmas
}

\section{$\operatorname{AUTHOR}(\mathrm{S}):$}

Fujii, K.; Mizushiri, K.; Nishioka, T.; Shikama, T.; Iwamae, A.; Goto, M.; Morita, S.; Kado, S.; Sawada, K.; Hasuo, $M$.

\section{CITATION:}

Fujii, K....[et al]. Development of multiwavelength-range fine-resolution spectrometer for hydrogen emissions and its application to large helical device periphery plasmas. REVIEW OF SCIENTIFIC INSTRUMENTS 2010, 81(3): 033106.

\section{ISSUE DATE:}

2010-03

URL:

http://hdl.handle.net/2433/147202

\section{RIGHT:}

Copyright 2010 American Institute of Physics. This article may be downloaded for personal use only. Any other use requires prior permission of the author and the American Institute of Physics. The following article appeared in REVIEW OF SCIENTIFIC INSTRUMENTS81, 033106 (2010) and may be found at 


\title{
Development of multiwavelength-range fine-resolution spectrometer for hydrogen emissions and its application to large helical device periphery plasmas
}

\author{
K. Fujii, ${ }^{1}$ K. Mizushiri, ${ }^{1}$ T. Nishioka,,${ }^{1}$ T. Shikama,${ }^{1}$ A. Iwamae,${ }^{2}$ M. Goto, ${ }^{3}$ S. Morita, ${ }^{3}$ \\ S Kado, ${ }^{4}$ K. Sawada, ${ }^{5}$ and M. Hasuo ${ }^{1}$ \\ ${ }_{1}^{1}$ Department of Mechanical Engineering and Science, Graduate School of Engineering, Kyoto University, \\ Kyoto 606-8501, Japan \\ ${ }^{2}$ Fusion Research and Development Directorate, Japan Atomic Energy Agency, Naka 311-0193, Japan \\ ${ }^{3}$ National Institute for Fusion Science, Toki 509-5292, Japan \\ ${ }^{4}$ Department of Nuclear Engineering and Management, School of Engineering, The University of Tokyo, \\ Tokyo 113-8656, Japan \\ ${ }^{5}$ Department of Applied Physics, Faculty of Engineering, Shinshu University, Nagano 380-8553, Japan
}

(Received 11 November 2009; accepted 15 February 2010; published online 26 March 2010)

We developed a spectrometer specialized for simultaneous observation of the hydrogen Balmer- $\alpha$, $-\beta,-\gamma$ lines and the Fulcher- $\alpha v^{\prime}=v^{\prime \prime}=2$ rovibronic transition band. The spectrometer was optimized for the light input coupled by nine optical fibers having $400 \mu \mathrm{m}$ core diameters. The spectral resolutions were $0.02-0.03 \mathrm{~nm}$ for these wavelength ranges at the entrance slit width of $20 \mu \mathrm{m}$. The polarization resolved spectra of these emissions from the peripheral region of large helical device (LHD) plasmas were measured simultaneously and showed the polarization dependence coming from the magnetic field in the LHD plasma. (C) 2010 American Institute of Physics.

[doi:10.1063/1.3356730]

\section{INTRODUCTION}

In a magnetically confined fusion plasma, dynamics of the neutral hydrogen and its isotopes in the peripheral region is known to have a strong influence on the core confinement. ${ }^{1,2}$ Spectroscopic diagnostics of the dynamics of the neutral hydrogen (or deuterium) has been proposed by many authors. ${ }^{3-7}$ From the observation of the line shape of the hydrogen Balmer- $\alpha$ emission from large helical device ${ }^{3}$ (LHD) and Torus of Research Institute for Applied Mechanics (TRIAM-1M), ${ }^{4}$ the emission locations of the atomic hydrogen in the peripheral region were determined from the Zeeman effect, together with the flow velocity and the temperature estimated from the Doppler effect. Regarding the molecular hydrogen, the Fulcher- $\alpha$ band spectra were measured for TRIAM-1M, and then the emission locations and the rotational and vibrational temperatures of hydrogen molecules were determined ${ }^{5}$ with a similar analysis to that of the hydrogen Balmer- $\alpha$ spectra. ${ }^{3,4}$ A simultaneous measurement of the line shapes of these spectra may provide the information about the neutral hydrogen transport as well as the dissociative dynamics of the molecules in the peripheral region.

The estimation of plasma parameters such as electron temperature and density in the peripheral region from the emission intensities of the Balmer series with identification of the emission locations may be valuable. Sawada et al. developed a collisional-radiative model for the system of hydrogen molecules and atoms in a plasma. ${ }^{8,9}$ They showed that the emission-line intensity ratios of the Balmer series show the dependence on the electron temperature, electron density, and the dissociative ratio. It is, however, difficult to measure high resolution line shapes and intensities of more than one wavelength range simultaneously with a conventional spectrometer. In this work, we developed a multiwavelength-range fine-resolution (MF) spectrometer for these hydrogen emissions and demonstrated it for the observation of neutral hydrogen in the peripheral region of LHD plasmas.

\section{MF SPECTROMETER}

Figure 1 shows a schematic illustration of the MF spectrometer. Emission from a plasma is introduced by optical fibers (core: $400 \mu \mathrm{m}$, cladding: $500 \mu \mathrm{m}, 24$ fibers are
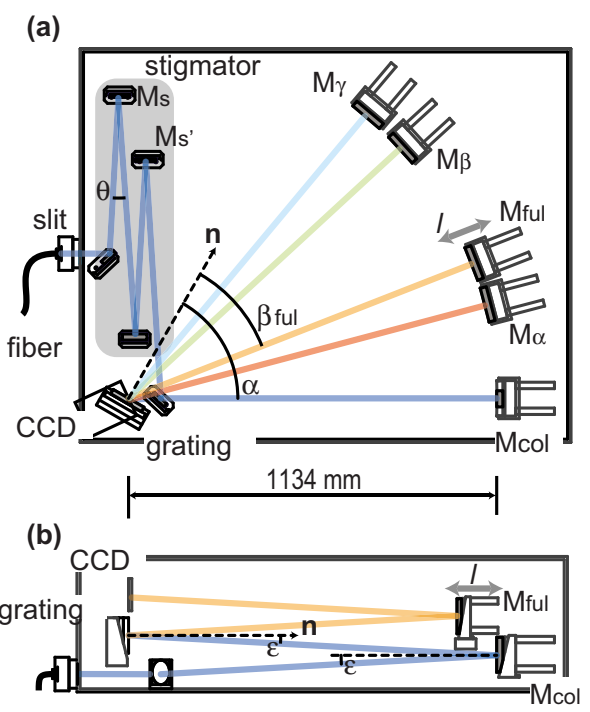

FIG. 1. (Color online) A schematic illustration of the MF spectrometer, (a) top and (b) side views. 
TABLE I. The diffraction angles $\beta$ at $\alpha=60^{\circ}$.

\begin{tabular}{lllll}
\hline \hline & $\mathrm{M}_{\alpha}$ & $\mathrm{M}_{\beta}$ & $\mathrm{M}_{\gamma}$ & $\mathrm{M}_{\text {ful }}$ \\
\hline$\beta$ [degree] & 45.3 & 17.6 & 10.2 & 39.0 \\
\hline \hline
\end{tabular}

bundled linearly with the center intervals of $500 \mu \mathrm{m})$, whose exits are located in front of the entrance slit. After passing through a stigmator, which is explained below, the introduced emission is collimated to be a parallel beam by a concave mirror ( $\mathrm{M}_{\mathrm{col}}$; focal length: $f=1143 \mathrm{~mm}$, diameter: $d=108 \mathrm{~mm})$. The parallel light beam is incident on a diffraction grating (2400 grooves $/ \mathrm{mm}$, ruled area: $102 \times 102 \mathrm{~mm}^{2}$ ). The diffracted light beams are focused on a charge coupled device (CCD) $\left(1024 \times 1024\right.$ pixels, $13 \times 13 \mu \mathrm{m}^{2} /$ pixel, Andor, DV435-BV) by four concave mirrors $\left(\mathrm{M}_{\alpha}, \mathrm{M}_{\beta}, \mathrm{M}_{\gamma}\right.$, $\left.\mathrm{M}_{\mathrm{ful}} ; f=1143 \mathrm{~mm}, d=108 \mathrm{~mm}\right)$ at the locations which correspond to the wavelengths of the Balmer- $\alpha(656 \mathrm{~nm}),-\beta$ $(486 \mathrm{~nm}),-\gamma(434 \mathrm{~nm})$, and the $Q$ branch of the Fulcher- $\alpha$ $v^{\prime}=v^{\prime \prime}=2$ rovibronic transition band $(620-622 \mathrm{~nm})$. Here, $v^{\prime}$ and $v^{\prime \prime}$ are the vibrational quantum numbers of the upper and lower states, respectively. The CCD detector is located just above the grating.

The diffraction angle $\beta$, at the incident angle $\alpha$, is calculated with the grating equation of

$$
\cos \varepsilon(\sin \alpha+\sin \beta)=m N \lambda,
$$

where $\alpha$ and $\beta$ are defined as shown in Fig. 1(a) in the plane perpendicular to the rulings of the grating (the vector normal to the grating surface is shown as $\boldsymbol{n}$ in Fig. 1.), while $\varepsilon$ is defined as the angle between the plane perpendicular to the rulings and the incident light beam as shown in Fig. 1(b). $N$, $m$, and $\lambda$ are the number of rulings per unit length, the diffraction order, and the wavelength of light, respectively. In our case, $m=1$.

The off-axis reflections at the concave mirrors cause comatic aberration and astigmatism. For the purpose of minimizing the aberrations, we set $\varepsilon$ to be $3^{\circ}$, which is the smallest angle determined from the size of the optical components we used. We set $\alpha$ to be $60^{\circ}$ to make enough separation between the diffraction angles of the observed emissions.
From Eq. (1), the diffraction angles $\beta$ for the Balmer- $\alpha,-\beta$, and $-\gamma$ lines and the Fulcher- $\alpha v^{\prime}=v^{\prime \prime}=2$ rovibronic transition band are calculated. The results are listed in Table I.

Even though the angle $\varepsilon$ is minimized, the term $\cos \varepsilon$ in Eq. (1) tilts the focused image of the fiber exits because the values of $\varepsilon$ are slightly different among the light passing through the different height of the slit. The magnitude of the tilt angle depends on the wavelength. We tilt the entrance slit together with the fiber bundle $2.0^{\circ}$ against the ruling direction of the grating to compensate this effect.

Because of the off-axis reflection at the concave mirrors, there is also some astigmatism on the focused image, which means the focused image on the CCD detector is expanded vertically. An example of this expansion is shown in Fig. 2(a). Since we use bundled fibers, it is demanded to reduce the vertical expansion. We compensate the astigmatism with a stigmator composed of two concave mirrors $\left(\mathrm{M}_{\mathrm{s}}, \mathrm{M}_{\mathrm{s}^{\prime}} ; f\right.$ $=635 \mathrm{~mm}, d=63.5 \mathrm{~mm}$ ) and one flat mirror, which are shown in the gray shadow in Fig. 1(a).

We designed the stigmator with a ray-tracing and multidimensional-minimization calculation assuming pure geometrical optics. We traced 100000 rays left from the five fiber exits aligned with a center distance of $500 \mu \mathrm{m}$. The fiber exits are located in front of the entrance slit with a width of $20 \mu \mathrm{m}$. We assumed that the rays leave uniformly from the fiber exits and calculated the image of the optical fiber exits focused on the CCD detector after reflections at the mirrors and diffraction at the grating. The condition in which the image was minimized horizontally and vertically was derived. The adjustable parameters for the minimization were the reflection angle $\theta$ of the stigmator and the focal adjustment length $l$ for $\mathrm{M}_{\text {ful }}$, the definitions of which are shown in Fig. 1.

Figure 2(b) shows the result of the calculation. The optimization is made for the light wavelength of $622 \mathrm{~nm}$, since the molecular emissions require high resolution because of their narrow Doppler widths. From the optimization, $\theta$ $=7.6^{\circ}$ and $l=0.36 \mathrm{~mm}$ were derived. The astigmatism is well compensated so that the images of the fiber exits are (a)

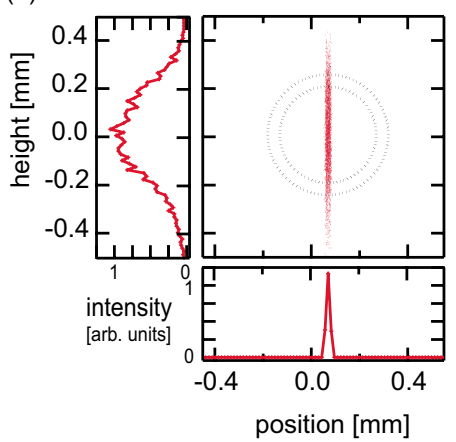

(b)

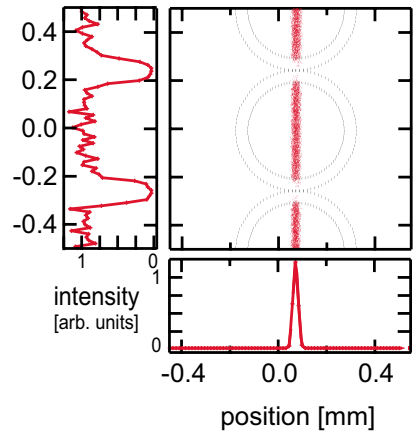

(c)

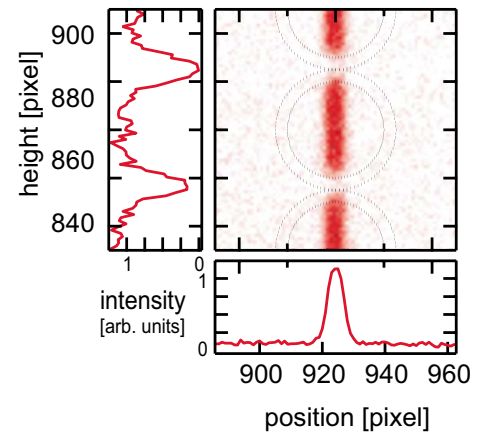

FIG. 2. (Color online) Calculated instrumental function of the MF spectrometer (a) without and (b) with the stigmator, and (c) the measured instrumental function with a thorium emission line of the wavelength $622.5 \mathrm{~nm}$ at the entrance slit width of $20 \mu \mathrm{m}$. The horizontal axis is parallel to the direction of the wavelength dispersion. The circles show the size of the core $(0.4 \mathrm{~mm})$ and the cladding $(0.5 \mathrm{~mm})$ of the optical fibers. The vertical and horizontal cross sections of the focused image are shown in the left and bottom sides, respectively. Although the scale of the axes in (a) and (b) are millimeters and that in (b) is pixels, the images can be relatively compared. The adjacent three images of the fiber exits are shown in (b) and (c), while only one image is shown in (a) for the purpose of avoiding the overlap of the images. 
(a)
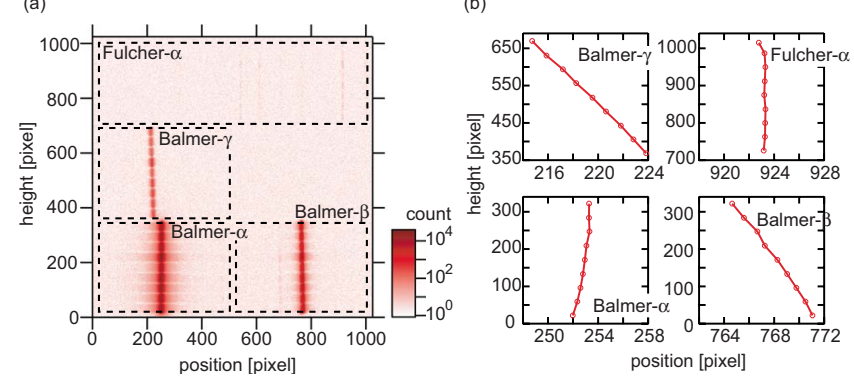

FIG. 3. (Color online) (a) A two dimensional image for the emission from a hydrogen low-pressure discharge tube focused on the CCD detector. The width of the entrance slit width is $20 \mu \mathrm{m}$. The area on which the image of each transition line is focused is closed by the dashed square. The nine bright spots aligned vertically in each area correspond to the nine fiber exits. Since the hydrogen molecular emission is very weak, it is hardly seen in the image. (b) The center positions of the bright spots.

clearly separated, and then we can use many optical fibers simultaneously and all the area of the CCD detector effectively.

Figure 2(c) shows the measured image of a thorium atomic emission line at the wavelength $622.5 \mathrm{~nm}$ from a thorium-argon hollow-cathode discharge lamp (Heraeus, P858A), the Doppler broadening of which is negligible. The measured full width at the half maximum in the horizontal direction $\left(\mathrm{FWHM}_{\text {mes }}\right)$ is 5.7 pixels $(105 \mu \mathrm{m})$, which is much larger than the calculation $\left(\mathrm{FWHM}_{\mathrm{cal}}=26 \mu \mathrm{m}\right)$. The broadening due to the diffraction limit of the grating, which is neglected in the ray-tracing calculation is smaller than $5 \mu \mathrm{m}$ and does not explain the discrepancy. Some aberration may be caused by insufficient accuracy in the locations of the optical components.

Figure 3(a) shows a CCD image for the emission from a hydrogen low-pressure discharge tube measured by the MF spectrometer. The areas, in which the images of the relevant transition lines are focused, are shown by dashed squares. We set vertical positions of Balmer- $\alpha$ and $-\beta$ lines to be the same on the CCD detector for the measurement with more optical fibers. We confirmed there are any other strong lines around $653-655 \mathrm{~nm}$ and $487-489 \mathrm{~nm}$ and the overlapped light from the other wavelength regions can be neglected for the measurement of the emission intensities and the line shapes. In this work, we can use the central nine fibers of the fiber bundle. The bright spots aligned vertically in each area correspond to the nine fiber exits at the entrance slit. The Fulcher- $\alpha$ band emission is hardly seen in the image within the scale of the figure because of their weak intensity. In Fig. 3(b), we show the center positions of the bright spots for the relevant hydrogen emissions. Since the entrance slit and the fiber bundle are tilted so as to align the spots of the Fulcher- $\alpha$ band emission vertically, the spots of other wavelength emissions tilt slightly. The tilt of every spots is, however, less than 2 pixels even for the Balmer- $\gamma$ emission of $430 \mathrm{~nm}$.

For the conversion from the horizontal pixels of an image such as Fig. 3(a) to the light wavelength, we used emission lines of the thorium-argon hollow-cathode discharge lamp which has many emission lines in the visible range. We obtained the emission spectra by binning the intensity in the
TABLE II. The instrumental widths of the MF spectrometer at the entrance slit width of $20 \mu \mathrm{m}$ for the hydrogen emission lines.

\begin{tabular}{ccccc}
\hline \hline & Balmer- $\alpha$ & Balmer- $\beta$ & Balmer- $\gamma$ & Fulcher- $\alpha v^{\prime}=v^{\prime \prime}=2$ \\
\hline FWHM[nm] & 0.019 & 0.023 & 0.030 & 0.022 \\
\hline \hline
\end{tabular}

CCD image over the vertical pixels for each spot. The instrumental widths at the entrance slit width of $20 \mu \mathrm{m}$ for the wavelength of the hydrogen emission lines are measured from the FWHM of the thorium atomic emissions and listed in Table II. We calibrated the absolute sensitivities of the MF spectrometer for each wavelength range using a standard lamp with a standard reflection sphere (Labsphere, USS600C).

\section{APPLICATION TO LHD PLASMAS}

We applied the MF spectrometer to the observation of LHD peripheral plasmas. The poloidal cross section of LHD and the lines of sight we used are shown in Fig. 4. We measured polarization resolved spectra with a polarization separation optics ${ }^{3}$ (PSO) because the spectral shapes are affected by the strong magnetic fields of LHD. The direction of the ordinary polarization of the PSO was set to be the magnetic direction at the inner ergotic layer.

An example of the spectra, which are measured simultaneously, is shown in Fig. 5. The spectra of ordinary ray (oray) are shown by the black lines while those of extraordinary ray (e-ray) are shown by the gray lines. The spectra were measured with an exposure time of $90 \mathrm{~ms}$, during a hydrogen discharge heated by the neutral beam injection at the total input power of $\sim 1.5 \mathrm{MW}$. The line-averaged electron density and temperature were $1.5 \times 10^{20} \mathrm{~m}^{-3}$ and 0.77 $\mathrm{keV}$, respectively. Observed intensities of the Balmer- $\alpha,-\beta$, $-\gamma$ and Fulcher $\alpha v^{\prime}=v^{\prime \prime}=2 Q 1$ spectra are 8.2, 1.3, 0.28, and $0.004 \mathrm{Wm}^{-2} \mathrm{sr}^{-1}$, respectively, and the intensity ratios are within the dynamic range of the CCD detector (16 bits). Note that the throughput of the spectrometer for each wavelength range can be controlled independently by adopting apertures on the corresponding focusing mirrors if necessary. The polarization dependence of the line shape is clearly observed for the Balmer- $\alpha$ line. The polarization dependence is also detectable for the Balmer- $\beta$ line and the $Q 1$ line of the Fulcher $\alpha v^{\prime}=v^{\prime \prime}=2$ rovibronic transition band. It is noticed that the polarization dependence of the intensities observed

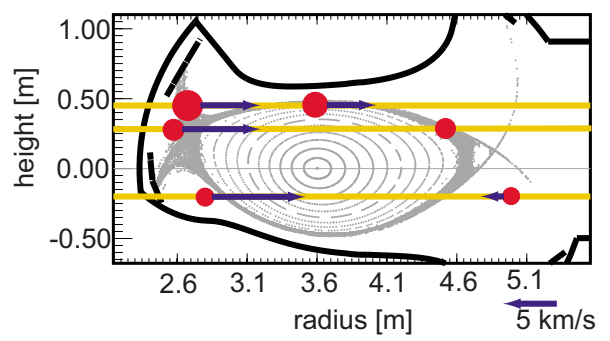

FIG. 4. (Color online) LHD poloidal cross section and the lines of sight for the spectra observation. The emission locations, intensities, and flow velocities derived from the observed Balmer- $\alpha$ spectra through the line shape analysis are indicated by the center of the circles, the area of the circles, and the length of the arrows, respectively. 


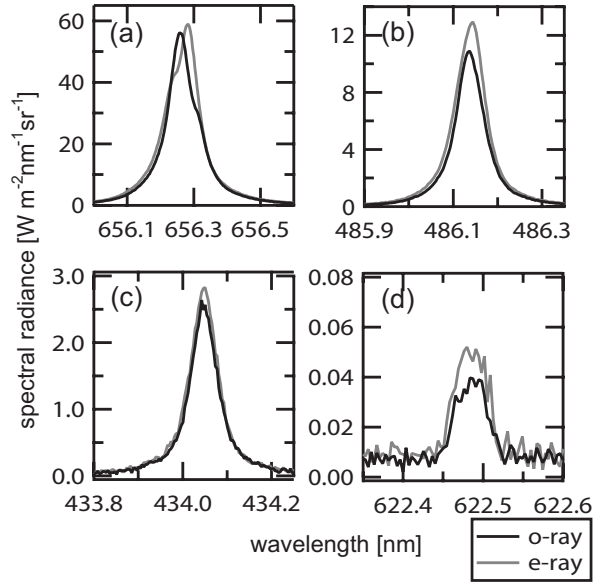

FIG. 5. An example of the hydrogen emission spectra from LHD plasma simultaneously observed with the MF spectrometer. The spectra of the Balmer- $\alpha,-\beta,-\gamma$ lines and the Fulcher $\alpha v^{\prime}=v^{\prime \prime}=2 Q 1$ line are shown in (a), (b), (c), and (d), respectively.

in Fig. 5, notably in (b) and (d), are due to the error of the sensitivity calibration of $\sim 15 \%$ at the present stage of the measurement.

We analyzed the observed Balmer- $\alpha$ line shapes by a least-squares fitting of the calculated Zeeman and Doppler profiles to both of the e-ray and o-ray line profiles. In the analysis, we assume that the positions of the Balmer- $\alpha$ emissions are localized two points on the line of sight (the inner and outer points) like the helium LHD plasma case. ${ }^{10}$ The velocity distribution of hydrogen atoms of each location is assumed to be expressed by a sum of three Maxwellian distributions. ${ }^{6}$ Details of the analysis method are described in Ref. 3. As shown in Fig. 6, the calculated spectra are well fitted to the observed ones. The emission locations, intensities, and flow velocities of hydrogen atoms derived from the fitting are shown in Fig. 4 by the centers of the circles, the areas of the circles, and the lengths of the arrows, respectively.

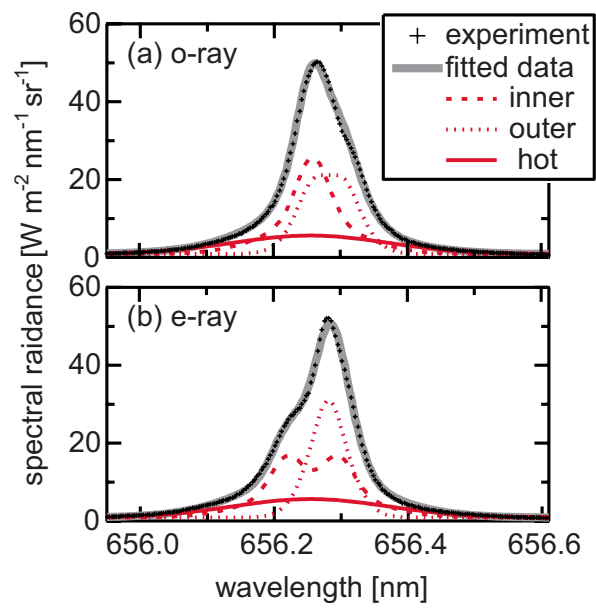

FIG. 6. (Color online) Fitted results (the thick gray lines) to the observed Balmer- $\alpha$ spectra (+). The o-ray and e-ray are shown in (a) and (b), respectively. The dashed, dotted, and solid lines show the inner, outer, and hot components, respectively.
We confirmed the line shapes of the other emissions to be consistent with calculated ones assuming the same emission locations derived from the Balmer- $\alpha$ line shape within the measurement accuracy. However, the present instrumental resolutions for the Balmer- $\beta$ and $-\gamma$ lines and the relatively bad signal to noise ratio of the Fulcher- $\alpha v^{\prime}=v^{\prime \prime}=2 Q 1$ line spectra were insufficient to separate the respective intensities at the two emission locations. In the case where the effect of the line integration for the emission observation can be neglected, this kind of measurement may be useful for the plasma diagnostics and the observation of the dynamics of neutral hydrogen.

\section{CONCLUSION}

We developed a spectrometer for simultaneous measurement of line shapes of the hydrogen Balmer- $\alpha,-\beta,-\gamma$ lines and the Fulcher- $\alpha$ band with the resolutions of $0.02-0.03 \mathrm{~nm}$ for the light input with nine optical fibers. With the spectrometer we observed polarization resolved emission spectra of these lines from the LHD plasma with the CCD detector exposure time of $90 \mathrm{~ms}$. Their emission intensities were 8.2, $1.3,0.28$, and $0.004 \mathrm{Wm}^{-2} \mathrm{sr}^{-1}$, respectively. The spectra showed the polarization dependence and allowed us the line profile analysis.

\section{ACKNOWLEDGMENTS}

This work was supported by the National Institute for Fusion Science (Grant No. NIFS08KOAP020).

${ }^{1}$ F. Wagner, G. Becker, K. Behringer, D. Campbell, A. Eberhagen, W. Engelhardt, G. Fussmann, O. Gehre, J. Gernhardt, G. v. Gierke, G. Haas, M. Huang, F. Karger, M. Keilhacker, O. Klüber, M. Kornherr, K. Lackner, G. Lisitano, G. G. Lister, H. M. Mayer, D. Meisel, E. R. Müller, H. Murmann, H. Niedermeyer, W. Poschenrieder, H. Rapp, H. Röhr, F. Schneider, G. Siller, E. Speth, A. Stäbler, K. H. Steuer, G. Venus, O. Vollmer, and Z. Yü, Phys. Rev. Lett. 49, 1408 (1982).

${ }^{2}$ N. Ohyabu, K. Narihara, H. Funaba, T. Morisaki, S. Masuzaki, K. Kawahata, A. Komori, O. Kaneko, H. Yamada, P. de Vries, M. Emoto, M. Goto, Y. Hamada, K. Ida, H. Idei, S. Inagaki, N. Inoue, S. Kado, S. Kubo, R. Kumazawa, T. Minami, J. Miyazawa, S. Morita, S. Murakami, T. Mutoh, S. Muto, Y. Nagayama, Y. Nakamura, H. Nakanishi, K. Nishimura, N. Noda, T. Kobuchi, S. Ohdachi, K. Ohkubo, Y. Oka, M. Osakabe, T. Ozaki, B. J. Peterson, A. Sagara, S. Sakakibara, R. Sakamoto, H. Sasao, M. Sasao, K. Sato, K. Saito, M. Sato, T. Seki, T. Shimozuma, M. Shoji, H. Suzuki, S. Sudo, Y. Takeiri, K. Tanaka, K. Toi, T. Tokuzawa, K. Tsumori, K. Tsuzuki, I. Yamada, S. Yamaguchi, K. Yamazaki, M. Yokoyama, K. Y. Watanabe, T. Watari, and O. Motojima, Phys. Rev. Lett. 84, 103 (2000).

${ }^{3}$ A. Iwamae, M. Hayakawa, M. Atake, T. Fujimoto, M. Goto, and S. Morita, Phys. Plasmas 12, 042501 (2005).

${ }^{4}$ T. Shikama, S. Kado, H. Zushi, M. Sakamoto, A. Iwamae, and S. Tanaka, Plasma Phys. Controlled Fusion 48, 1125 (2006).

${ }^{5}$ T. Shikama, S. Kado, H. Zushi, and S. Tanaka, Phys. Plasmas 14, 072509 (2007).

${ }^{6}$ J. D. Hey, C. C. Chu, Ph. Mertens, S. Brezinsek, and B. Unterberg, J. Phys. B 37, 2543 (2004).

${ }^{7}$ H. Kubo, H. Takenaga, T. Sugie, S. Higashijima, S. Suzuki, A. Sakasai, and N. Hosogane, Plasma Phys. Controlled Fusion 40, 1115 (1998).

${ }^{8}$ K. Sawada, K. Eriguchi, and T. Fujimoto, J. Appl. Phys. 73, 8122 (1993).

${ }^{9}$ K. Sawada and T. Fujimoto, J. Appl. Phys. 78, 2913 (1995).

${ }^{10}$ M. Goto and S. Shigeru, Phys. Rev. E 65, 026401 (2002). 